Artigo / Article

\title{
Indicações para início de tratamento na leucemia linfóide crônica
}

\author{
Chronic lymphocytic leukemia: When to start treatment
}

Carlos S. Chiattone

Na leucemia linfóide crônica (LLC-B), o número de linfócitos é geralmente maior ou igual a $5.000 / \mathrm{mm}^{3}$, com imunofenótipo característico (células B co-expressando o antígeno CD5). ${ }^{1}$ Como os métodos laboratoriais têm se tornado mais sensíveis na detecção de células monoclonais no sangue periférico, hoje se identifica uma situação chamada "linfocitose monoclonal de significado indeterminado" (em analogia à gamopatia monoclonal de significado indeterminado).

Esta linfocitose monoclonal incipiente pode ser observada em até $3 \%$ dos indivíduos maiores de 40 anos e em cerca de $6 \%$ dos maiores de 60 anos. $^{2}$ A conseqüência imediata desta detecção cada vez mais freqüente é, do ponto de vista epidemiológico, uma falsa impressão do aumento da sobrevida para este grupo de pacientes. As conseqüências para o paciente, que eventualmente poderia passar o resto de sua vida sem nenhuma manifestação clínica desta alteração, são a de gerar desconforto emocional sem motivo, mas principalmente a de poder resultar em tratamento desnecessário, como conseqüência de eventual procedimento médico açodado. Estas situações são cada vez mais comuns na medicina atual, onde são utilizadas mais freqüentemente as avaliações clínicas periódicas (check-up), acompanhadas de aumento da sensibilidade dos exames laboratoriais. Estes achados inesperados podem ser chamados, em uma livre denominação, de "pseudo-doença" ou "sobrediagnóstico".

Como os tratamentos com doses convencionais atualmente disponíveis não são curativos, a terapia antileucêmica é freqüentemente desnecessária na doença inicial não complicada, ${ }^{3}$ especialmente nesses casos diagnosticados ao acaso em indivíduos assintomáticos.

Disciplina de Hematologia e Oncologia,FCM da Santa Casa de São Paulo.

Hemocentro da Santa Casa de São Paulo.

Correspondência para: Carlos S. Chiattone

Faculdade de Ciências Médicas da Santa Casa de São Paulo, Disciplina de Hematologia e Oncologia. Hemocentro da Santa Casa de São Paulo.

Rua Marquês de Itu, 579

01223-001 - São Paulo - SP, Brasil

E-mails:dir.bs@santacasasp.org.br; carlos.chiattone@terra.com.br 
Deve-se ainda levar em conta, na decisão de se iniciar o tratamento, que a LLC-B ocorre predominantemente em pacientes na idade média e em idosos, aumentando sua incidência em décadas sucessivas. ${ }^{4}$ A mediana de sobrevida, baseada em estudos das décadas de 70 e 80 , tem sido estimada entre 8 e 12 anos. $^{3}$

O curso clínico da doença progride desde linfocitose indolente, sem outros comemorativos, até infiltração de órgãos linfóides como linfonodos, fígado, baço e medula óssea, com conseqüente linfonodomegalia, esplenomegalia, hepatomegalia e citopenia no sangue periférico. Complicações da(s) citopenia(s) incluem anemia, responsável por comprometimento da qualidade de vida, além de infecção e sangramento, que representam as principais causas de morte de pacientes com LLC-B. ${ }^{5}$

Alterações imunológicas como teste de Coombs direto positivo (com ou sem anemia hemolítica), púrpura trombocitopênica imunológica e redução dos níveis de imunoglobulinas podem ser responsáveis por manifestações clínicas ou mesmo dificultar o tratamento da LLC-B. ${ }^{6}$ Estas alterações devem ser investigadas tanto ao diagnóstico como periodicamente, durante o seguimento do paciente, e são geralmente indicações de início de tratamento.

Além dos clássicos, como os sistemas de estadiamento de Rai e de Binet, novos fatores prognósticos têm sido identificados como possíveis indicadores do perfil de evolução, como: subgrupo de alteração citogenética, estado mutacional da imunoglobulina, expressão da ZAP-70 e expressão do CD38. ${ }^{7}$ Os novos fatores prognósticos serão abordados em outro artigo deste fascículo da RBHH.

Antes de analisar o tratamento a ser instituído, é importante que o diagnóstico seja confirmado. Devem ser excluídas doenças que, clínica ou laboratorialmente, podem se assemelhar à LLC-B, como o linfoma de célula do manto (que também expressa concomitantemente CD20 e CD19 com CD5), a tricoleucemia, a macroglobulinemia de Waldenström, a leucemia prolinfocítica e a leucemia linfocítica de grandes linfócitos granulosos, tanto de fenótipo T, quanto NK. Cerca de $1 \%$ de casos com morfologia mais variável, mas muitas vezes indistinguível da LLC, expressam marcadores T (CD4 e CD7) e têm rearranjo clonal de genes de receptores de célula T. Estes pacientes têm freqüentemente lesões cutâneas, sobrevida mais curta e mínima resposta à quimioterapia. ${ }^{9} \mathrm{~A}$ nova classificação da OMS para doenças linfoproliferativas inclui estes casos entre as leucemias prolinfocíticas $\mathrm{T}$.

O tratamento da LLC-B abrange desde a observação periódica do paciente (procedimento de watch and wait), sem medicação específica, até uma variedade de opções terapêuticas. Estas incluem: corticosteróides, agentes alquilantes, análogos de purina, quimioterapia combinada, anticorpos monoclonais, quimioimunoterapia e transplante. ${ }^{10}$ Por ser doença incurável, incidir preferentemente em idosos e progredir lentamente, a LLC-B é geralmente tratada conservadoramente. ${ }^{11}$
Em pacientes assintomáticos, o tratamento pode ser adiado até que o paciente se torne sintomático, na progressão da doença. Como a taxa de progressão varia de paciente a paciente, por vezes com longos períodos de estabilidade e até com regressão espontânea da doença, é necessário um seguimento freqüente e cuidadoso para monitorar a evolução clínica.

Uma metanálise de estudos randomizados comparando, em pacientes com estágio inicial da LLC-B, tratamento imediato com tratamento adiado até ocorrência de progressão da doença, não mostrou diferença na sobrevida global. ${ }^{12}$

Antes de iniciar o tratamento, considerações devem ser feitas em relação ao paciente, à doença e ao tratamento:

1) Fatores relacionados ao paciente, como idade, performance status, comorbidades e aspectos culturais, psicológicos e filosóficos do paciente.

2) Fatores relacionados à doença, como presença e intensidade dos sintomas e presença de fatores prognósticos adversos.

3) Fatores relacionados ao tratamento, como contraindicações, efeitos colaterais e, quando da recidiva, duração da resposta a tratamentos anteriores.

4) Considerações fármaco-econômicas são particularmente importantes em países com nossas características de desenvolvimento socioeconômico.

O manuseio da LLC-B é dificultado pela heterogeneidade da idade do paciente na apresentação (cada vez mais se diagnosticam pacientes mais jovens), bem como pela variabilidade na história natural da doença, como atestada pelos diferentes fatores prognósticos. A mediana de sobrevida de pacientes com doença avançada é normalmente superior ao observado na maioria das outras doenças hematológicas neoplásicas e dos tumores sólidos.

Aproximadamente um terço dos pacientes jamais necessitarão de tratamento, morrendo de causas não relacionadas à LLC-B; em outro terço, a fase inicial indolente é seguida de progressão da doença; o terço remanescente tem doença agressiva desde o início, necessitando de terapia imediata. ${ }^{15}$

Os critérios clássicos aceitos para se iniciar o tratamento são ainda os destacados pelo guidelines de LLC do grupo de trabalho patrocinado pelo Instituto Nacional de Câncer dos EUA (NCI-WG) publicado em 1996. ${ }^{14}$ Recentemente, durante o XI International Workshop on CLL, realizado em Nova York, EUA em setembro de 2005, foi relatado que no novo guidelines, prestes a ser finalizado, pouco será alterado nos critérios para início do tratamento enfatizados no guidelines de 1996. (Michael J. Hallek, informação pessoal)

Um subgrupo de pacientes é considerado portador de LLC-B indolente (smoldering CLL). Tem as seguintes características: estádio 0 de Rai (ou A de Binet), medula óssea 
com padrão de envolvimento não difuso, valor da hemoglobina igual ou superior a $13,0 \mathrm{~g} / \mathrm{dL}$, número de linfócitos no sangue periférico inferior a $30.000 / \mathrm{mL}$ e tempo de duplicação de linfócitos igual ou menor que 12 meses. ${ }^{16,17}$ Com o conhecimento dos novos fatores prognósticos, este subgrupo de pacientes com LLC-B indolente pode ser caracterizado como: Rai 0 ou Binet A expressando genes de Ig mutados e sem alterações cromossômicas negativas. ${ }^{18}$ Este grupo corresponde a cerca de $40 \%$ das LLC-B. ${ }^{18}$ A estes pacientes, em particular, não se deve iniciar terapia até que haja clara progressão da doença. Muitos destes pacientes transcorrerão toda sua vida sem necessidade de tratamento.

Outros pacientes recém-diagnosticados com estádio inicial (Rai 0 ou 1, Binet A) também devem ser monitorados, sem tratamento, até evidente progressão da doença. Dois estudos, um europeu, do grupo francês cooperativo em LLC, ${ }^{19}$ e outro dos EUA, do grupo CALGB,${ }^{20}$ confirmaram que, em estágios iniciais, o tratamento imediato não prolonga a sobrevida global. Estes estudos foram, no entanto, realizados utilizando-se tratamento baseado em agentes alquilantes. O papel do tratamento com análogos de purina, anticorpos monoclonais e suas associações em estágios iniciais ainda é desconhecido.

Contrariamente, há consenso que pacientes com doença mais avançada (estádios B e C de Binet ou III e IV de Rai) ou com doença menos avançada mas progressiva, grupos cuja expectativa de vida não excede sete anos, devam ser tratados precocemente. ${ }^{18}$

Deve-se lembrar que os pacientes com doença avançada, com comorbidade importante, dependendo das condições clínicas, podem ser apenas observados (watch and wait) até que ocorra uma clara evidência de progressão ou aparecimento de sintomas.

Diversas manifestações caracterizam doença ativa, indicando necessidade de tratamento tanto em estudos clínicos, como na prática clínica. ${ }^{14,15} \mathrm{O}$ início da terapia é recomendado para os seguintes grupos de pacientes:

1. Anemia e/ou trombocitopenia (estádios III ou IV de Rai; estádio C de Binet)

2. Sintomas relacionados à doença, como fadiga, sudorese noturna, perda de peso, febre e linfonodomegalia dolorosa.

3. Doença progressiva, como demonstrada pelo aumento da linfocitose (linfocitose progressiva com aumento de $>50 \%$ em dois meses ou tempo antecipado de duplicação de linfócitos menor que seis meses) e/ou aumento rápido de linfonodos, baço ou fígado. Contrariamente, linfonodomegalia localizada e transitória, em decorrência de infecção local, não é necessariamente indicação de tratamento.

4. Anemia e/ou trombocitopenias auto-imunes com resposta insatisfatória a coticosteróides.

5. Embora hipogamaglobulinemia, mesmo que acen- tuada, não seja necessariamente indicação de terapia, infecção de repetição é indicação para início de tratamento. Entretanto, não é claro o benefício dos tratamentos disponíveis para esta complicação.

É importante discutir quanto ao número de linfócitos no sangue periférico. Diferente de outras leucemias, sintomas relacionados à leucoestase são raros na LLC-B. Portanto, o número de linfócitos, mesmo que expressivo, não é, por si só, indicativo de início de tratamento. Alguns autores consideram $100.000 / \mathrm{mm}^{3}$ o valor acima do qual poder-seia indicar início do tratamento, mas há controvérsia quanto a este aspecto.

Em resumo, a decisão de tratar o paciente deve ser guiada pelo estádio clínico, pela presença de sintomas e pela atividade da doença. Apenas os pacientes com doença avançada (Rai III e IV ou Binet B ou C) mostram vantagem no início imediato do tratamento. Pacientes com doença inicial (Rai 0-II ou Binet A) geralmente não são tratados imediatamente após o diagnóstico, sendo monitorados com a estratégia de watch and wait. $\mathrm{O}$ tratamento para os estádios iniciais é indicado apenas quando houver presença de sintomas relacionados à doença ou sinais de alta atividade da LLC-B.

Por último, deve sempre ser lembrado que os critérios de início, bem como os de interromper e os de reintroduzir o tratamento são, de certa forma, vagos, devendo sempre, principalmente na prática clínica, ser individualizados ao perfil do paciente.

\begin{abstract}
In spite of the great advances made in understanding its biology, chronic lymphocytic leukemia (CLL-B) is still incurable. Historically, the objective of the treatment of CLL-B has been to alleviate the disease-related symptoms and to prolong the survival. Chemotherapy for all patients in the initial stages of the disease does not obtain an increase in the survival, but rather, only causes an unnecessary toxicity. The decision to treat the patient is oriented by the clinical conditions, by the presence of symptoms and by the signs of activity of the disease. With the conventional treatments currently available, there is only evidence of an advantage in immediate treatment, directly following the diagnosis, for patients in an advanced stage (Rai III and IV or Binet B and C). Patients in initial stages (Rai 0-II or Binet A) generally should not receive immediate treatment, but only be periodically observed. In the initial stages, treatment is only indicated if disease-related symptoms (such as: B symptoms, reduction in the performance status, or organomegaly symptoms or complications) or signs of highly active CLL-B (such as: lymphocyte duplication time of less than six months or a rapid growth in lymph nodes) are present. Rev. bras. hematol. hemoter. 2005;27(4):272-275.
\end{abstract}

Key Words: Chronic lymphocytic leukemia; treatment. 


\section{Referências Bibliográficas}

1. Dighiero G. Chronic lymphocytic leukemia biology and prognosis. Hematology 2005;278- 284.

2. Rawstron AC, Yuille MR, Fuller J et al. Monoclonal B lymphocytes with the caracteristics of indolent"chronic lymphocytic leukemia are presente in 3,5\% of adults with normal blood counts. Blood 2002; 100(2):635-9.

3. Rozman C, Montserrat E. Chronic lymphocytic leukemia. N Engl J Med 1995;333(16):1.052-7.

4. Catovsky D, Fooks J, Richards S. Prognostic factors in chronic lymphocytic leukemia: the importance of age, sex and response to treatment in survival.A report from the MRC CLL 1 trial. MRC Working Party on Leukemia in Adults. Br J Haematol 1989;72(2):141-9.

5. Anaissie EJ, Kontoyiannis DP, O'Brien S et al. Infections in patients with chroni lymphocytic leukemia treated with fludarabine. Ann Inter Med 1998;129(7):559-66

6. Mauro FR, Foa R, Cerretti R et al. Autoimmune hemolytic anemia in chronic lymphocytic leukemia: clinical, therapeutic and prognostic features. Blood 2000; 95(9):2.786-92

7. Chiorazzi N, Rai KR, Ferrarini M. Chronic lymphocytic leukemia. N Engl J Med 2005;352(8):804-15

8. Tsimberidou AM, Keating MJ. Richter syndrome: biology, incidence, and therapeutic strategies. Cancer 2005;103(2):216-8.

9. Hoyer JD, Ross CW, Li CY et al. True T-cell chronic lymphocytic leukemia: a morphologic and immunophenotypic study of 25 cases. Blood 1995; 86(3):1.163-9

10. Keating MJ. Chronic lymphocytic leukemia. Semin Oncol 1999;26(5 Suppl 14): $107-4$

11. Faguet GB: Chronic lymphocytic leukemia: an updated review. J Clin Oncol 1994;12(9):1.974-90

12. Chemotherapeutic options in chronic lymphocytic leukemia: a metaanalysis of the randomized trial. CLL Trialists' Collaborative Group. J Natl Cancer Inst 1999;91(10):861-8
13. Dighiero G, Binet JL. When and How to treat chronic lymphocytic leukemia. NEJM 2000;343(24):1.799-801.

14. Cheson BD, Bennett JM, Grever M et al. National Cancer Institute Sponsored Working Group Guidelines for Chronic Lymphocytic Leukemia: Revised Guidelines for Diagnosis and Teatment. Blood 1996;87(12):4.990-97

15. Chronic lymphocytic leukemia: recommendations for diagnosis, staging, and response criteria. International Workshop on Chronic Lymphocytic Leukemia. Ann Intern Med 1989;110:236.

16. Natural history of stage A chronic lymphocytic leukaemia untreated patients. French Cooperative Group on Chronic Lymphocytic Leukaemia. Br J Haematol 1990;76(1):45-57.

17. Molica S. Progression and survival studies In early chronic lymphocytic leukemia. Blood 1991;78(4):895-9.

18. Dighiero G Perspectives in chronic lymphocytic leukemia: biology and management. Hematol Oncol Clin N Am 2004;18: 927-43.

19. A randomized clinical trial of chlorambucil versus COP in stage B chronic lymphocytic leukemia. The French Cooperative Group on Chronic Lymphocytic Leukemia. Blood 1990;75(7):1.422-5

20. Shustik C, Mick R, Silver R et al. Treatment of early chronic lymphocytic leukemia: Intermittent chlorambucil versus observation. Hematol Oncol 1998;6(1):7-12.

Avaliação: Carlos Sergio Chiattone

(publicado após acordo do Editor)

Conflito de interesse: Artigo derivado do II Encontro Brasileiro de

Consenso da LLC.

Recebido: 23/01/2006

Aceito: 23/01/2006 